\section{IDDF2019-ABS-0216 CLINICAL OUTCOMES AFTER PLACEMENT OF COLONIC METALLIC STENTS IN PATIENTS WITH LARGE BOWEL OBSTRUCTION}

Toh Yoon Ezekiel Wong*, Yohei Kubota, Haruna Nakamura, Kazuki Nishihara. ${ }^{1}$ Hiroshima Kyoritsu Hospital, Japan

\subsection{6/gutjnl-2019-IDDFabstracts.199}

Background Placement of colonic metallic stents (CMS) for malignant large bowel obstruction either as a bridge to surgery (BTS) or best supportive care (BSC) has been covered by national health insurance since 2012 in Japan. When possible, CMS is often considered a preferable intervention in cases deemed unsuitable for surgical resection. In this retrospective study, we report our experience with CMS, focusing on the improvement in oral intake levels.

Methods 20 patients (13 men and 7 women) who developed large bowel obstruction and received CMS placements at our hospital from 2014 to 2019 were included for analysis. Baseline characteristics such as age, gender, type of obstruction and tumor location were reviewed. Intervention success rate was defined as having a ColoRectal obstruction scoring system (CROSS) level of 4 after CMS placement.

Results Patient's average age was 74 (53 to 101) years old. Cause of bowel obstruction were malignancies in 19 patients and benign inflammation (diverticulitis) in one patient. Tumor (or obstruction) was located in the transverse colon in 3 patients (15\%), descending colon in 4 patients (20\%), sigmoid colon in 6 patients $(30 \%)$ and rectum in 7 patients $(35 \%)$. Intervention success rate was $90 \%(18 / 20)$. Length of CMS used was $60 \mathrm{~mm}$ in 5 patients, $80 \mathrm{~mm}$ in 8 patients, $100 \mathrm{~mm}$ in 4 patients and $120 \mathrm{~mm}$ in 4 patients. One patient required double stenting (stent in stent) due to insufficient bowel dilation after initial CMS placement. The aim of CMS was BTS in 14 patients and BSC in 6 patients. The mean CROSS level was 2.9 prior to stenting and 3.7 (including intervention failures) after stenting. The average survival after CMS placement in BSC patients was 122 (4 to 360) days. In BTS patients, the average time to surgery was 23 (16 to 42$)$ days.

Conclusions Placement of CMS is an effective method to alleviate large bowel obstruction and avoid emergency decompression surgery. When the intervention is successful, CMS can improve oral intake and is a feasible option in patients unable to undergo surgery due to various risk factors, including old age.

\section{IDDF2019-ABS-0223 SMALL BOWEL ULTRASOUND FINDING OF RECURRENT COLIC ABDOMEN IN PERITONEAL (DRY TYPE) TUBERCULOSIS}

\footnotetext{
${ }^{123}$ Maria Goretti Ametembun*. 'Saumlaki, Tanimbar, West of South-East Moluccas; ${ }^{2}$ St Elisabeth Hospital - Lela, Flores, Nusa Tenggara Timur; ${ }^{3}$ Stela Maris and Lukas Hilisimetano
} Hospitals- South Nias - North Sumatera, Indonesia

\subsection{6/gutjn|-2019-IDDFabstracts.200}

Background Number of patients with recurrent colic abdominal pain without clear diagnostic, even many patients had very severe abdominal pain entered in hospitals several times without exact diagnostic in these at least health facilities.

Objective To describe small bowel ultrasound finding of recurrent colic abdomen pain of peritoneal (dry type) tuberculosis.
Methods This descriptive study conducted at some rural boundary islands: Saumlaki, Tanimbar, Moluccas (February-June 2010), St Elisabeth Hospital, Flores, (February 2012 -August 2013) and Stela Maris-Lukas Hilisimetano Hospitals South Nias-North Sumatera (April-July 2015). Patients whom diagnosed as peritoneal (dry type) tuberculosis clinically, based on sub-acute/chronic recurrent colic abdominal history, accompanied by diarrhea/constipation, weight loss, loss of appetite, chronic recurrent low-grade fever, night sweats, fatigue and malabsorption symptoms; doughy abdomen with dam-board phenomena according to peritoneal dry type tuberculosis; high blood sedimentation rate and lymphocyte increased; and the X-ray shown pulmonary tuberculosis. Small bowel ultrasound examined with convex probe $3.5-6 \mathrm{MHz}$ as the only available ultrasound equipment at that time by one certified ultrasound internal medicine specialist. Small bowel wall thickness (of 80 patients) in the dullness pain area were compared within the tympanic area.

Results N=1929 adult, >15-78y (Saumlaki 329, Flores 1299, South Nias 301). The recurrent colic abdominal pain with tenderness were associated with doughy and dull area of damboard phenomena of peritoneal tuberculosis. No tenderness on tympanic area. Ultrasound finding in the dullness area were hypoperistaltic, thickening heterogenic hypoechoic of the small bowel wall, loss differentiation and irregular margin of the wall in addition to several round nodular structures (patchy hyper echoic non-shadowing with an irregular hypoechoic rim) within the wall and narrowing of the lumen. The mean of small bowel wall thickness in the dull area were $(6,2-22,6$ $\mathrm{mm}$, mean $12,44 \mathrm{~mm}(2,63 \mathrm{x})$ compared with in tympanic area were $(3,7-4,8 \mathrm{~mm}$, mean $4,6 \mathrm{~mm})$.

Conclusions Small bowel ultrasound finding of the recurrent colic abdomen was associated with thickening of small bowel wall and narrowing of its lumen in the dull area of damboard phenomena in peritoneal (dry type) tuberculosis.

\section{IDDF2019-ABS-0226 THE POTENTIAL INTESTINAL FUNGAL BIOMARKERS IN PATIENTS WITH COLONIC POLYPS}

${ }^{1}$ Qiongyun Chen*, 'Bangzhou Zhang, ${ }^{1}$ Hongzhi Xu, ${ }^{1}$ Jianlin Ren, ${ }^{2}$ Xiang Zhang. 'Zhongshan Hospital Department of Gastroenterology, Xiamen University, China; ${ }^{2}$ Institute for Microbial Ecology, Medical College of Xiamen University, China

\subsection{6/gutjn|-2019-IDDFabstracts.201}

Background Epidemiological data show that at least $80 \%$ of colorectal cancers evolved from colon polyps, and early intervention can greatly reduce the risk of colorectal cancer. However, the cost of a colonoscopy is high and the acceptance is low, which causes the patient to miss the opportunity of diagnosis and treatment. Therefore, exploring new and effective colon polyps screening methods is of great significance for the early diagnosis and treatment of colorectal cancer.

Methods We conducted a randomized controlled trial in Zhongshan Hospital Affiliated to Xiamen University, Xiamen, China. We recruited volunteers with normal hematology and colonoscopy as controls. Patients with polyps were diagnosed with colonoscopy and histopathology. Volunteer's feces were subjected to Internal Transcribed Spacer (ITS) analysis for fungi, and the microbial community structure changes were investigated by R-analysis method.

Results 12 controls and 17 patients were enrolled in our project. The results showed that compared with the control, the 